\title{
MRI-Based Texture Features as Potential Prognostic Biomarkers in Anaplastic Astrocytoma Patients Undergoing Surgical Treatment
}

\author{
Yang Zhang, ${ }^{1,2}$ Chaoyue Chen $\mathbb{D}^{1,2}$ Yangfan Cheng, ${ }^{2}$ Danni Cheng, ${ }^{2}$ Fumin Zhao $\mathbb{D},^{3}$ \\ and Jianguo $\mathrm{Xu} \mathbb{D}^{1,2}$ \\ ${ }^{1}$ Department of Neurosurgery, West China Hospital, Sichuan University, Chengdu, China \\ ${ }^{2}$ West China School of Medicine, West China Hospital, Sichuan University, Chengdu, China \\ ${ }^{3}$ Department of Radiology, West China Second University Hospital, Sichuan University, Chengdu, China
}

Correspondence should be addressed to Fumin Zhao; hxzfm@sina.com and Jianguo Xu; drjianguoxu@gmail.com

Received 14 September 2019; Revised 26 April 2020; Accepted 19 May 2020; Published 12 June 2020

Academic Editor: André L. B. de Barros

Copyright (C) 2020 Yang Zhang et al. This is an open access article distributed under the Creative Commons Attribution License, which permits unrestricted use, distribution, and reproduction in any medium, provided the original work is properly cited.

\begin{abstract}
Objectives. The purpose of this study was to investigate whether texture features from magnetic resonance imaging (MRI) were associated with the overall survival (OS) of anaplastic astrocytoma (AA) patients undergoing surgical treatment. Methods. A total of 51 qualified patients who were diagnosed with AA and underwent surgical interventions in our institution were enrolled in this retrospective study. Patients were followed up for at least 30 months or until death. Texture features derived from histogram-based matrix (HISTO) and grey-level co-occurrence matrix (GLCM) were extracted from preoperative contrast-enhanced T1-weighted images. Each texture feature was dichotomized based on its optimal cutoff value calculated by receiver operating characteristics curve analysis. Kaplan-Meier analysis and log rank test were conducted to compare the 30-month OS between the dichotomized subgroups. Multivariate Cox regression analysis was performed to determine independent prognostic factors. Results. Three HISTO-derived features (HISTO-Energy, HISTO-Entropy, and HISTO-Skewness) and five GLCM-derived features (GLCMContrast, GLCM-Energy, GLCM-Entropy, GLCM-Homogeneity, and GLCM-Dissimilarity) were found to be significantly correlated with 30-month OS. Moreover, GLCM-Homogeneity $(p=0.001$, hazard ratio $=6.351)$ was suggested to be the independent predictor of the patient survival. Conclusion. MRI-based texture features have the potential to be applied as prognostic biomarkers in AA patients undergoing surgical treatment.
\end{abstract}

\section{Introduction}

Anaplastic astrocytoma (AA) is a malignant brain tumor with aggressive nature and high risk of progression to glioblastoma, accounting for $6.7 \%$ of primary central nervous system glioma based on the data from Central Brain Tumor Registry of the United States [1]. The first-line therapy of AA is the maximum safe resection followed by possibly radiotherapy and chemotherapy based on the condition of patients and genetic status of tumors [2]. Despite the advancement of neurosurgical techniques, radiotherapy and chemotherapy regimen, the five-year relative survival rate for patients with AA is still poor at 19.8\% [1].
Magnetic resonance (MR) scan is considered as the most common examination for AA patients in clinical practice, but few studies have extracted the in-depth information from this routinely obtained image and explored its prognostic values.

Texture analysis has been widely utilized to extract quantitative statistics from medical images and provide nonvisual information related to the pathophysiology of lesions [3]. Previous studies have demonstrated that texture features extracted from magnetic resonance imaging (MRI) represented feasible ability in prognosis prediction for a variety of tumors, such as breast cancer, nasopharyngeal carcinoma, hepatocellular carcinoma, glioblastoma, and 
ovarian cancer [4-8]. Moreover, histogram-based matrix (HISTO) and grey-level co-occurrence matrix (GLCM) were the most commonly reported matrices and were suggested to be associated with the patient survival [9-12]. However, there has been no study exploring the prognostic value of MRI-based texture features in patients with AA. Therefore, the purpose of the present study was to investigate whether texture features derived from HISTO and GLCM could serve as new prognostic factors in AA patients undergoing surgical treatment.

\section{Methods}

2.1. Patient Selection. In this retrospective study, we screened our institutional database to review the patients who were diagnosed and treated at the neurosurgery department of our institution from May 2013 to June 2016. We initially selected the potentially eligible patients (a) who were with pathological confirmation of AA; (b) who had a history of undergoing maximum surgical resection of AA; and (c) who had available preoperative MR images. And, the patients were excluded if they were (a) had a history of receiving any antitumor therapies such as radiotherapy, chemotherapy, or radiosurgery before MR scans; (b) with clinical evidence of postoperative hemorrhage, infection, or other diseases that might reduce the patient survival significantly; and (c) with incomplete medical records or follow-up information. It was worth noting that we also excluded those patients who died within the six months after surgery considering their death might result from surgical complications like infection and hemorrhage. Clinical parameters of qualified patients were recorded, including gender, age, history of receiving postoperative radiotherapy and chemotherapy (temozolomide-based regimen), and isocitrate dehydrogenase (IDH) status of tumors. Overall survival (OS) calculated from the date of MR scan to the date of tumor-related death or the last follow-up (30 months after surgery) was taken as the endpoint of this study. This study was approved by the institutional ethics review board. The written informed consent was obtained from all the participants.

2.2. MRI Acquisition. MR scans were conducted in our institutional radiology department using the 3.0 T GE SIGNA MRI scanner. The sequences included T1-weighted image, T2-weighted image, and contrast-enhanced T1weighted (T1C) image with gadopentetate dimeglumine $(0.1 \mathrm{mmol} / \mathrm{kg})$ as the contrast agent. The parameters of $\mathrm{T} 1 \mathrm{C}$ sequence were as follows: time repetition $=1600 \mathrm{~ms}$, time echo $=9.2 \mathrm{~ms}$, slice thickness $=5 \mathrm{~mm}$, and field of view $=240 \times 240 \mathrm{~mm}^{2}$.

2.3. Texture Feature Extraction. Texture features were extracted by two researchers together using LIFEx software under the guidance of a senior radiologist [13]. Only T1C sequence was chosen for feature extraction considering its relatively clear depiction on the boundary of tumors compared with other sequences. After drawing the regions of interest (ROI) within the border of tumors on each layer of axial image, three-dimensional texture features could be automatically generated by the software. Disagreements on the border of tumors between two researchers were addressed by consulting the senior radiologist. A total number of ten features from two commonly reported matrices were selected for further analysis, including Skewness, Kurtosis, Entropy, Energy from HISTO, Homogeneity, Entropy, Energy, Dissimilarity, Correlation, and Contrast from GLCM [9-12]. Explanations and formulas of the above texture parameters are shown in Supplementary Material 1.

2.4. Statistical Analysis. All statistical analyses in the current study were performed using IBM SPSS Statistics for Windows (Version 22.0, IBM Corp. Armonk, NY, USA) and MedCalc statistics (MedCalc Software, Belgium). One-way analysis of variance (ANOVA) test was conducted to identify the effects of the baseline clinical characteristics on the patient survival. Considering the complicated relationship among texture features, Pearson's correlation between each pair of feature was evaluated. Receiver operating characteristic (ROC) analysis was performed on each texture feature to calculate the $p$ value, area under the curve (AUC), 95\% confidence interval (CI), and optimal cutoff values (defined at the maximal Youden's index). Then, each texture feature was dichotomized based on its optimal cutoff values. Kaplan-Meier (KM) analysis and log rank test were conducted to assess whether texture features were significantly associated with the 30-month OS. The significant texture features and clinical parameters were included as input variables for multivariate Cox regression analysis (method: forward LR) to determine which factor could be taken as the independent predictor of the patient survival. The statistics would be considered significant if the $p$ value was less than 0.05 . The workflow of this study is shown in Figure 1.

\section{Results}

3.1. Characteristics of Patients. A total number of 51 eligible patients were enrolled in this study. The mean age was 39.75 years, and the gender ratio was about $3: 2$ (male : 30, female: $21)$. Thirty-eight patients $(74.51 \%)$ undertook postoperative radiotherapy, and twenty-seven patients (52.94\%) received postoperative temozolomide-based chemotherapy. IDH mutation of the tumor was observed in thirty-one patients (60.78\%). The results of one-way ANOVA test indicated that IDH status of the tumor, postoperative radiotherapy, and chemotherapy were significantly related to the patient survival. In accordance with the previous studies, patients who were diagnosed with IDH-mutant AA and received postoperative radiotherapy and chemotherapy represented better prognosis $[14,15]$. Within 30 months after surgery, tumor-related death was observed in 21 patients $(41.18 \%)$, and 30 patients (58.82\%) were still alive in the last follow-up. Detailed characteristics of patients are shown in Table 1.

3.2. OS Prediction. Most texture features were positively or negatively correlated with each other according to Pearson's correlation (Figure 2). ROC analysis demonstrated that three 


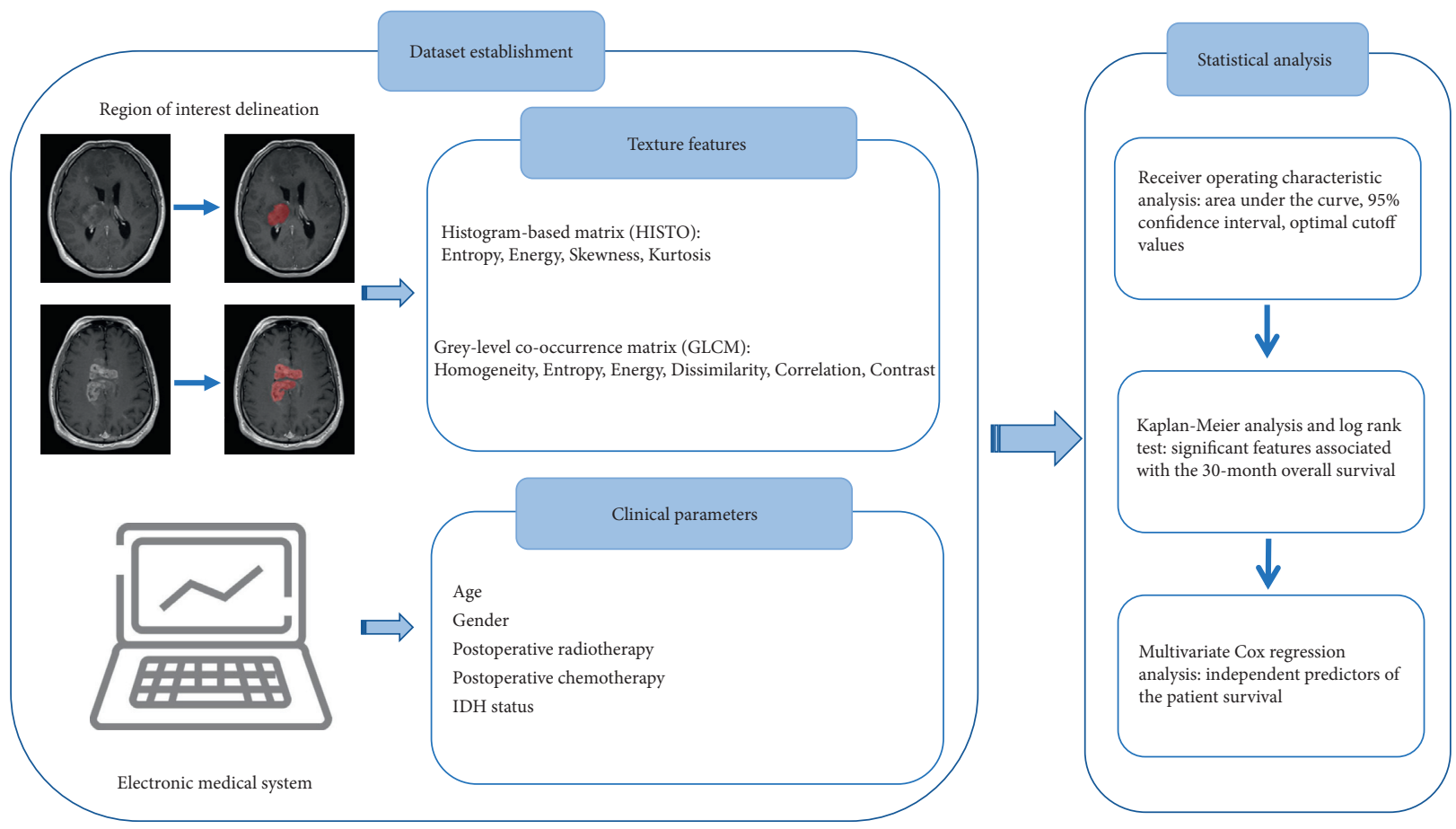

Figure 1: The flow chart of the present study.

TABLE 1: Characteristics of patients.

\begin{tabular}{lcc}
\hline Characteristic & Value & $p$ value \\
\hline Age (year) & & \\
$\quad$ Mean \pm SD & $39.75 \pm 16.97$ & 0.752 \\
$\quad$ Range & $7-69$ & \\
\hline $\begin{array}{l}\text { Gender, } n(\%) \\
\quad \text { Male }\end{array}$ & $30(58.82)$ & - \\
$\quad$ Female & $21(41.18)$ & \\
\hline Postoperative radiotherapy, $n(\%)$ & $38(74.51)$ & 0.002 \\
$\quad$ Yes & $13(25.49)$ & \\
$\quad$ No & & \\
\hline Postoperative chemotherapy, $n(\%)$ & & \\
$\quad$ Yes & $27(52.94)$ & 0.019 \\
$\quad$ No & $24(47.06)$ & \\
\hline IDH status, $n$ (\%) & $31(60.78)$ & 0.028 \\
$\quad$ IDH-mutant & $20(39.22)$ & \\
$\quad$ IDH-wildtype &
\end{tabular}

$\mathrm{SD}$, standard deviation; IDH, isocitrate dehydrogenase.

texture features from HISTO (HISTO-Energy, HISTO-Entropy, and HISTO-Skewness) and five texture features from GLCM (GLCM-Contrast, GLCM-Dissimilarity, GLCMEnergy, GLCM-Entropy, and GLCM-Homogeneity) were found to be significantly associated with the patient survival. Detailed statistics of each feature including its optimal cutoff value, AUC, 95\% CI were listed in Table 2. KM analysis and log rank test demonstrated significantly better 30 -month OS in patients with higher GLCM-Contrast $(p=0.002)$, higher GLCM-Dissimilarity $(p=0.002)$, lower GLCM-Energy $(p=0.024)$, higher GLCM-Entropy $(p=0.005)$, lower GLCM-Homogeneity $(p<0.001)$, lower HISTO-Energy ( $p=0.006)$, higher HISTO-Entropy $(p=0.006)$, and lower
HISTO-Skewness $(p<0.001)$. Multivariate Cox proportional hazards regression analysis indicated that GLCMHomogeneity $(p=0.001$, hazard ratio $(\mathrm{HR})=6.351)$ and postoperative radiotherapy $(p=0.001, \mathrm{HR}=0.223)$ could be taken as the independent predictor of 30-month OS for AA patients (Table 3). The KM survival curve of GLCMHomogeneity is shown in Figure 3.

\section{Discussion}

In the current study, we investigated the prognostic value of texture features extracted from T1C sequence of MRI in AA patients who had undertaken surgical treatment. A series of texture features were suggested to be significantly associated with the 30-month OS. Moreover, GLCM-Homogeneity was found to be the independent prognostic predictor of the patient survival. Given that the T1C sequence is routinely conducted for AA patients in clinical practice, MRI-based texture features could potentially serve as feasible prognostic biomarkers to facilitate clinical decision-making.

From the perspective of precision medicine, accurate survival prediction is essential for clinicians to make the personalized therapeutic decision for cancer patients. For patients with $\mathrm{AA}$, the age and performance status of the patient were considered to be important therapy-independent prognostic factors, while the extent of resection and receipt of adjuvant chemotherapy and radiotherapy were taken as therapy-dependent prognostic factors $[2,15]$. Besides, some molecular markers were reported to be useful in survival prediction, such as IDH mutation which was often associated with better prognosis in AA patients $[16,17]$. However, the detection of IDH status through either immunohistochemistry or sequencing requires additional 


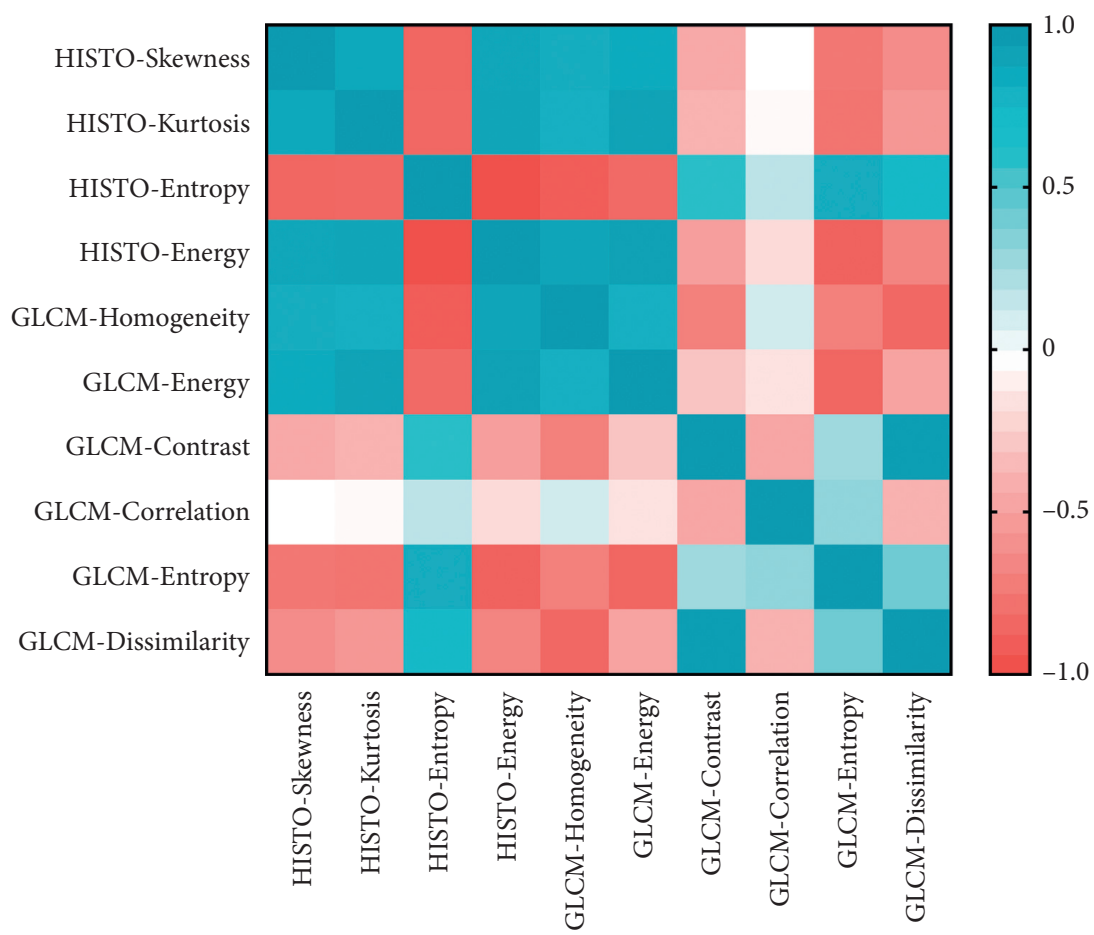

Figure 2: Heat map showing Pearson's correlations between all pairs of texture features.

TABLE 2: Values of significant texture features in receiver operating characteristic analysis.

\begin{tabular}{lcccc}
\hline Texture features & $p$ value & AUC & $95 \%$ CI & Optimal cutoff value \\
\hline HISTO-Energy & 0.022 & 0.679 & 0.534 to 0.803 & 0.035 \\
HISTO-Entropy & 0.025 & 0.676 & 0.531 to 0.800 & 1.540 \\
HISTO-Skewness & $<0.001$ & 0.767 & 0.627 to 0.874 & 0.360 \\
GLCM-Contrast & 0.013 & 0.692 & 0.547 to 0.814 & 77.446 \\
GLCM-Dissimilarity & 0.004 & 0.716 & 0.572 to 0.833 & 6.421 \\
GLCM-Energy & $<0.001$ & 0.757 & 0.617 to 0.866 & 0.002 \\
GLCM-Entropy & 0.022 & 0.678 & 0.532 to 0.802 & 2.755 \\
GLCM-Homogeneity & $<0.001$ & 0.808 & 0.673 to 0.905 & 0.257 \\
\hline
\end{tabular}

HISTO, histogram-based matrix; GLCM, grey-level co-occurrence matrix; AUC, area under the curve; CI, confidence interval.

TABLE 3: Multivariate Cox proportional hazard regression analysis of texture and clinical parameters.

\begin{tabular}{lccc}
\hline Parameters & $p$ value & HR & $95 \%$ CI \\
\hline GLCM-Homogeneity & 0.001 & 6.351 & $2.097 \sim 19.228$ \\
Postoperative radiotherapy & 0.001 & 0.223 & $0.090 \sim 0.550$ \\
\hline
\end{tabular}

HISTO, histogram-based matrix; GLCM, grey-level co-occurrence matrix; $\mathrm{HR}$, hazard ratio; $\mathrm{CI}$, confidence interval.

platform and cost, and the results could only be obtained a few days after surgical interventions. MR scan is the most common, noninvasive examination for patients with AA, so extracting prognostic information and survival predictors from the routinely performed MRI could be clinically applicable.

Texture analysis, which could quantify the gray-level patterns of lesions in medical images, has been widely applied in recent researches. Several studies indicated significant associations between texture parameters derived from MRI and patients prognosis in many types of tumors
[18-21]. Specifically, one study reported that texture features extracted from preoperative MRI were related to survival of endometrial cancer patients [18]. Another study on clinically nonfunctioning pituitary adenoma suggested that MRIderived texture parameters were correlated with tumor recurrence or progression [19]. As for glioma, the most common brain tumor, most studies focused on glioblastoma patients, whereas the prognostic value of MRI-based texture features in AA patients had rarely been explored [6, 22-24]. Therefore, in the present study, we analyzed texture features from two representative matrices and built a set of new prognostic biomarkers through routinely performed T1C sequence. Our results suggested that GLCM-Homogeneity was the independent predictor of the 30-month OS in AA patients, and lower GLCM-Homogeneity value $(<0.257)$ was associated with better prognosis. GLCM-Homogeneity reflects the homogeneity of grey-level voxel pairs and has also been found to be correlated with the patient survival in the previous studies [25-27]. One study reported that GLCMHomogeneity was the significant predictor of both overall 


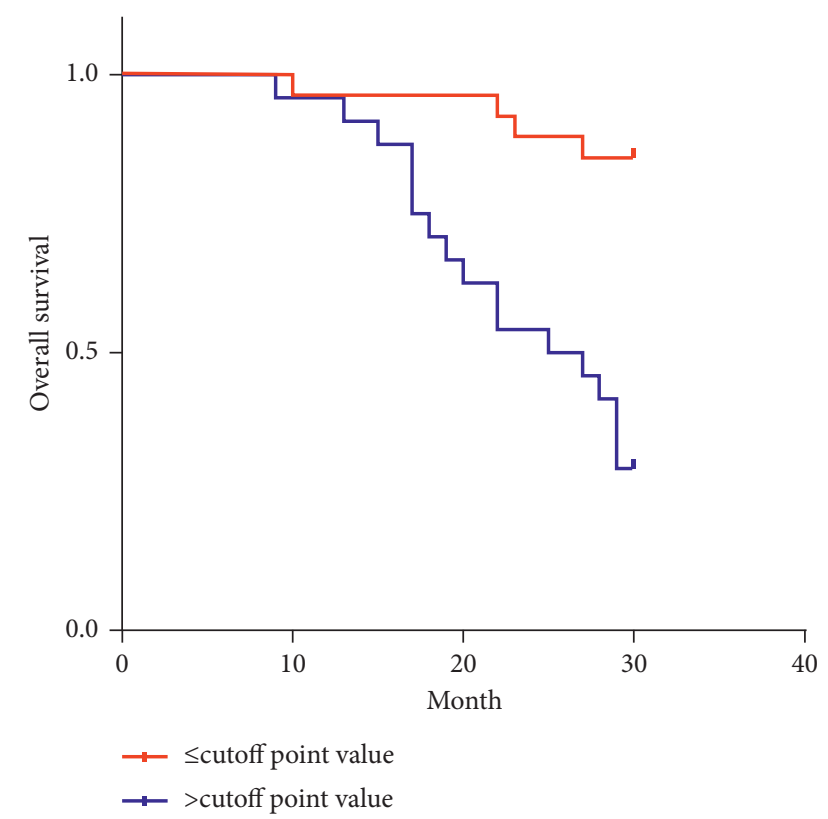

FIgURE 3: Kaplan-Meier curve of overall survival based on GLCMHomogeneity at 30 months. Kaplan-Meier curve shows that the lower GLCM-Homogeneity value $(p<0.001)$ is associated with better patient survival.

survival and disease-free survival in patients with resectable lung cancer [26]. Another study on cervical cancer reported that GLCM-Homogeneity was the independent predictor of lymph node metastasis that was the important determinant of the patient survival [27]. Similar to our results, this study also found increased GLCM-Homogeneity value within the tumor was correlated with relatively poorer prognosis. Although the underlying mechanism was still unclear, one study had indicated that tumors with higher GLCM-Homogeneity values represented poorer response to chemotherapy and radiotherapy, which might provide a potential explanation for our results [28]. Future studies are required to investigate the specific mechanism of this feature as the prognostic predictor. Besides, texture features were found to represent different predictive performances. Considering the relatively small sample size in this study, researches with larger patient cohort are warranted to verify our results.

The ability of texture analysis in prognosis prediction may result from its association with the underlying histopathology of the tumor, such as cellular density, intratumoral heterogeneity, and vascular microenvironment, which had been demonstrated to be linked to tumor proliferation, progression, and therapeutic response $[11,29-31]$. Besides, several studies had characterized the correlation between texture parameters and the genetic status of tumors. Specifically, one study on rectal cancer suggested that MRI-based texture feature could be used to predict the mutation status of Kirsten Ras (KRAS), an important oncogene associated with the patient survival [32]. Furthermore, in glioma, texture features from MRI were able to predict the IDH status that was a recognized prognostic factor [33]. The conventional approach of assessing the biological and genetic characteristics of AA through pathological examination could only be conducted after surgical interventions and may be limited in representing characteristics of the entire tumor when the tumor cannot be completely removed in some cases. In this context, texture analysis from routine MRI could be a potential option to make the systematic assessment of tumors preoperatively and predict the prognosis of patients and guide further treatment. However, the specific relationship between texture parameters and pathophysiological process within the tumor seems to be complicated and requires more researches in the future.

There were some limitations in the current study. First, as a retrospective study, the possibility of selection bias cannot be excluded. Second, texture analysis was only conducted on routine $\mathrm{T1C}$ sequence in this study, requiring more researches to explore the prognostic value of features from advanced MR sequences. Third, we only analyzed texture features derived from histogram-based matrix and grey-level co-occurrence matrix since they were two of the most common matrices in the previous studies [9-12]. Future studies were needed to investigate the prognostic value of features from other matrices. Fourth, our results were limited by the small datasets from a single institution and should be validated in multicenter studies with the larger sample size.

\section{Conclusion}

In conclusion, texture features from routine MRI were associated with 30-month OS in AA patients undergoing surgical treatment and could potentially serve as prognostic predictors. Larger prospective studies are required to validate our results and investigate the prognostic value of texture features further.

\section{Data Availability}

The data used to support the findings of this study are available from the corresponding author upon request.

\section{Ethical Approval}

All procedures performed in the studies involving human participants were in accordance with the ethical standards of the institutional and/or national research committee and with the 1964 Helsinki Declaration and its later amendments or comparable ethical standards. In this retrospective study, the institutional review board approved this retrospective study.

\section{Conflicts of Interest}

The authors declare that they have no conflicts of interest.

\section{Authors' Contributions}

Yang Zhang and Chaoyue Chen contributed equally to this work. 


\section{Acknowledgments}

This work was supported by 1.3.5 Project for Disciplines of Excellence, West China Hospital, Sichuan University (ZYJC18007), and Key Research and Development Project of Science and Technology Department of Sichuan Province (2019YFS0392).

\section{Supplementary Materials}

Supplementary Material 1: Explanations and formulas of texture parameters. (Supplementary Materials)

\section{References}

[1] Q. T. Ostrom, H. Gittleman, G. Truitt, A. Boscia, C. Kruchko, and J. S. Barnholtz-Sloan, "CBTRUS statistical report: primary brain and other central nervous system tumors diagnosed in the United States in 2011-2015," Neuro-oncology, vol. 20, no. suppl_4,pp. iv1-iv86, 2018.

[2] M. Weller, M. van den Bent, J. C. Tonn et al., "European association for neuro-oncology (EANO) guideline on the diagnosis and treatment of adult astrocytic and oligodendroglial gliomas," The Lancet Oncology, vol. 18, no. 6, pp. e315-e329, 2017.

[3] P. Lambin, R. T. H. Leijenaar, T. M. Deist et al., "Radiomics: the bridge between medical imaging and personalized medicine," Nature Reviews Clinical Oncology, vol. 14, no. 12, pp. 749-762, 2017.

[4] J.-H. Kim, E. S. Ko, Y. Lim et al., "Breast cancer heterogeneity: MR imaging texture analysis and survival outcomes," Radiology, vol. 282, no. 3, pp. 665-675, 2017.

[5] B. Zhang, J. Tian, D. Dong et al., "Radiomics features of multiparametric MRI as novel prognostic factors in advanced nasopharyngeal carcinoma," Clinical Cancer Research, vol. 23, no. 15, pp. 4259-4269, 2017.

[6] M. Ingrisch, M. J. Schneider, D. Nörenberg et al., "Radiomic analysis reveals prognostic information in T1-weighted baseline magnetic resonance imaging in patients with glioblastoma," Investigative Radiology, vol. 52, no. 6, pp. 360-366, 2017.

[7] H. Zhang, Y. Mao, X. Chen et al., "Magnetic resonance imaging radiomics in categorizing ovarian masses and predicting clinical outcome: a preliminary study," European Radiology, vol. 29, no. 7, pp. 3358-3371, 2019.

[8] X.-H. Wang, L.-H. Long, Y. Cui et al., "MRI-based radiomics model for preoperative prediction of 5-year survival in patients with hepatocellular carcinoma," British Journal of Cancer, vol. 122, no. 7, pp. 978-985, 2020.

[9] A. Hocquelet, T. Auriac, C. Perier et al., "Pre-treatment magnetic resonance-based texture features as potential imaging biomarkers for predicting event free survival in anal cancer treated by chemoradiotherapy," European Radiology, vol. 28, no. 7, pp. 2801-2811, 2018.

[10] H. Park, Y. Lim, E. S. Ko et al., "Radiomics signature on magnetic resonance imaging: association with disease-free survival in patients with invasive breast cancer," Clinical Cancer Research, vol. 24, no. 19, pp. 4705-4714, 2018.

[11] K. Nie, L. Shi, Q. Chen et al., "Rectal cancer: assessment of neoadjuvant chemoradiation outcome based on radiomics of multiparametric MRI," Clinical Cancer Research, vol. 22, no. 21, pp. 5256-5264, 2016.
[12] F. Lucia, D. Visvikis, M.-C. Desseroit et al., "Prediction of outcome using pretreatment 18F-FDG PET/CT and MRI radiomics in locally advanced cervical cancer treated with chemoradiotherapy," European Journal of Nuclear Medicine and Molecular Imaging, vol. 45, no. 5, pp. 768-786, 2018.

[13] C. Nioche, F. Orlhac, S. Boughdad et al., "LIFEx: a freeware for radiomic feature calculation in multimodality imaging to accelerate advances in the characterization of tumor heterogeneity," Cancer Research, vol. 78, no. 16, pp. 4786-4789, 2018.

[14] S. Chang, P. Zhang, J. G. Cairncross et al., "Phase III randomized study of radiation and temozolomide versus radiation and nitrosourea therapy for anaplastic astrocytoma: results of NRG Oncology RTOG 9813," Neuro-oncology, vol. 19, no. 2, pp. 236-258, 2017.

[15] J. Y. Shin and A. Z. Diaz, "Anaplastic astrocytoma: prognostic factors and survival in 4807 patients with emphasis on receipt and impact of adjuvant therapy," Journal of Neuro-Oncology, vol. 129, no. 3, pp. 557-565, 2016.

[16] M. J. van den Bent, M. Smits, J. M. Kros, and S. M. Chang, "Diffuse infiltrating oligodendroglioma and astrocytoma," Journal of Clinical Oncology, vol. 35, no. 21, pp. 2394-2401, 2017.

[17] M. Weller, S. M. Pfister, W. Wick, M. E. Hegi, G. Reifenberger, and R. Stupp, "Molecular neuro-oncology in clinical practice: a new horizon," The Lancet Oncology, vol. 14, no. 9, pp. e370-e379, 2013.

[18] S. Ytre-Hauge, J. A. Dybvik, A. Lundervold et al., "Preoperative tumor texture analysis on MRI predicts high-risk disease and reduced survival in endometrial cancer," Journal of Magnetic Resonance Imaging, vol. 48, no. 6, pp. 1637-1647, 2018.

[19] B. P. Galm, E. L. Martinez-Salazar, B. Swearingen et al., "MRI texture analysis as a predictor of tumor recurrence or progression in patients with clinically non-functioning pituitary adenomas," European Journal of Endocrinology, vol. 179, no. 3, pp. 191-198, 2018.

[20] A. Bhatia, M. Birger, H. Veeraraghavan et al., "MRI radiomic features are associated with survival in melanoma brain metastases treated with immune checkpoint inhibitors," Neuro-oncology, vol. 21, no. 12, pp. 1578-1586, 2019.

[21] J. Fang, B. Zhang, S. Wang et al., "Association of MRI-derived radiomic biomarker with disease-free survival in patients with early-stage cervical cancer," Theranostics, vol. 10, no. 5, pp. 2284-2292, 2020.

[22] Y. Liu, X. Xu, L. Yin, X. Zhang, L. Li, and H. Lu, "Relationship between glioblastoma heterogeneity and survival time: an MR imaging texture analysis," American Journal of Neuroradiology, vol. 38, no. 9, pp. 1695-1701, 2017.

[23] K. Chang, B. Zhang, X. Guo et al., "Multimodal imaging patterns predict survival in recurrent glioblastoma patients treated with bevacizumab," Neuro-oncology, vol. 18, no. 12, pp. 1680-1687, 2016.

[24] S. Bae, Y. S. Choi, S. S. Ahn et al., "Radiomic MRI phenotyping of glioblastoma: improving survival prediction," Radiology, vol. 289, no. 3, pp. 797-806, 2018.

[25] V. Nardone, P. Tini, C. Nioche et al., "Texture analysis as a predictor of radiation-induced xerostomia in head and neck patients undergoing IMRT," La Radiologia Medica, vol. 123, no. 6, pp. 415-423, 2018.

[26] J. Choe, S. M. Lee, K.-H. Do et al., "Prognostic value of radiomic analysis of iodine overlay maps from dual-energy computed tomography in patients with resectable lung cancer," European Radiology, vol. 29, no. 2, pp. 915-923, 2019. 
[27] W.-C. Shen, S.-W. Chen, J.-A. Liang, T.-C. Hsieh, K.-Y. Yen, and C.-H. Kao, "[18] fluorodeoxyglucose positron emission tomography for the textural features of cervical cancer associated with lymph node metastasis and histological type," European Journal of Nuclear Medicine and Molecular Imaging, vol. 44, no. 10, pp. 1721-1731, 2017.

[28] F. Tixier, C. C. Le Rest, M. Hatt et al., "Intratumor heterogeneity characterized by textural features on baseline 18FFDG PET images predicts response to concomitant radiochemotherapy in esophageal cancer," Journal of Nuclear Medicine, vol. 52, no. 3, pp. 369-378, 2011.

[29] S. Y. Ahn, C. M. Park, S. J. Park et al., "Prognostic value of computed tomography texture features in non-small cell lung cancers treated with definitive concomitant chemoradiotherapy," Investigative Radiology, vol. 50, no. 10, pp. 719-725, 2015.

[30] C. Su, J. Jiang, S. Zhang et al., "Radiomics based on multicontrast MRI can precisely differentiate among glioma subtypes and predict tumour-proliferative behaviour," European Radiology, vol. 29, no. 4, pp. 1986-1996, 2019.

[31] N. Fujima, A. Homma, T. Harada et al., "The utility of MRI histogram and texture analysis for the prediction of histological diagnosis in head and neck malignancies," Cancer Imaging, vol. 19, no. 1, p. 5, 2019.

[32] J. E. Oh, M. J. Kim, J. Lee et al., "Magnetic resonance-based texture analysis differentiating KRAS mutation status in rectal cancer," Cancer Research and Treatment, vol. 52, no. 1, pp. 51-59, 2020.

[33] H. Zhou, K. Chang, H. X. Bai et al., "Machine learning reveals multimodal MRI patterns predictive of isocitrate dehydrogenase and $1 p / 19 q$ status in diffuse low- and high-grade gliomas," Journal of Neuro-Oncology, vol. 142, no. 2, pp. 299-307, 2019. 